\title{
Quasi-optical lens antenna made of electromagnetic-induction materials
}

\author{
Jinbang Wang ${ }^{1, ~ a}$, Hanxue Mei, b ${ }^{1,}$ Zhiguo Liu ${ }^{1,2, c^{*}}$ and Tao Zhang, 2, d \\ ${ }^{1}$ Key Laboratory for Beam Technology and Materials Modification of Ministry of Education, Beijing \\ Normal University, Beijing, China \\ ${ }^{2}$ Beijing Radiation Center, Beijing, China \\ a271336192@qq.com, b827265298@qq.com, ${ }^{\text {c, }}{ }^{*}$ liuzhigu0512@126.com, daozhang@bnu.edu.cn
}

Keywords: Microwave, Lens antenna, Imaging system, Refraction

Abstract. A biconvex lens was designed and fabricated by embedding electromagnetic-induction materials (cage-shaped granules of conductor materials, CGC) in polymethyl methacrylate (PMMA). The CGC material has a refractive index of 1.504 at $35 \mathrm{GHz}$. The spatial resolution and depth of focus of the active millimeter wave (AMMW) imaging system were analyzed. Millimeter-wave (MMW) images of a square and two bars have been obtained with the biconvex lens. The image quality was acceptable, proving that the CGC material has the ability to refract MMWs in MMW imaging.

\section{Introduction}

MMW imaging techniques have many potential applications, such as security check in airports, remote sensing, and aircraft landing guidance [1-3]. For security checkpoints, AMMW imaging is better because of its high sensitivity and signal-to-noise ratio. In an AMMW system, using the focusing element to control the size of the beam is vitally important. Usually, a lens is chosen to control the size of the beam because it's easier to design in the near field and easier to manufacture than a reflector [4-5]. The performance of the focusing lens used in AMMW system directly affects the image quality. So far, the focusing lenses for MMW focal plane imaging are mainly made of polymer materials, such as Rexolite, polyethylene and Teflon [6-8]. However, lenses made of polymer material have not been widely used in practical applications [3].

The refractive mechanism of electromagnetic induction and the concept of refractive energy were presented in [9]. This theory has been used to calculate the refractive indices of $\mathrm{He}, \mathrm{Ne}$ and $\mathrm{Ar}$, and good verifications have been achieved [10-12]. The CGC material has been designed; see Fig. 1[3, 13]. CGC has many closed loops of conductors through which the magnetic field of an electromagnetic wave can go to produce strong interactions of electromagnetic induction in CGC.
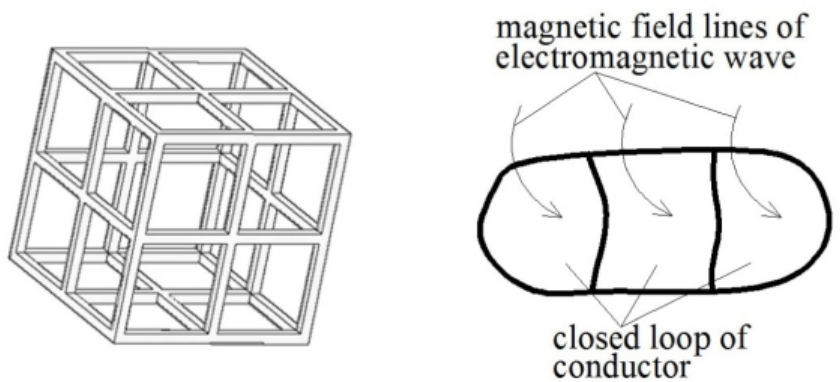

Figure 1. Schematic of CGC

In this paper, we design and fabricate a biconvex lens by embedding CGC materials in PMMA materials. The effective focal length of the biconvex lens is measured. Millimeter-wave (MMW) images of a square and two bars have been obtained with the biconvex lens.

\section{Design and measurement of the biconvex lens}

Dielectric lens design is mainly based on Fermat's principle, i.e., the principle of least time. A better result is obtained in designing the lens antenna when the optical axis feed has no optical path difference as the starting point [14]. Since the lens design is based on geometric optics, the aperture size of the 
lens must be sufficiently large, preferably more than 20 wavelengths, to make the geometric optics assumption valid [15]. The biconvex lens with an aperture diameter $D=200 \mathrm{~mm}$ was designed and fabricated by CGC materials embedded in PMMA, see Fig. 2.

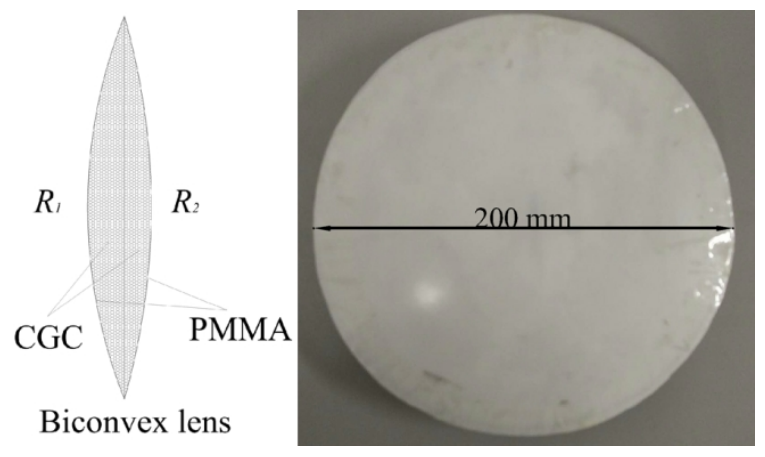

Figure 2. Schematic and photo of biconvex lens

We measured the refractive index $n$ of CGC materials at $35 \mathrm{GHz}$, and $n=1.504$. Biconvex lens can be seen as two plano-convex lenses. Generally, a spherical Plano-convex lens introducing the minimum aberration-limited blurring performs nearly the best form lens due to its shape factor of $1[3,16]$. For this consideration, we used two plano-convex lenses with radii of curvature of 270 and $400 \mathrm{~mm}$ to achieve the effect of a biconvex lens.

The combination of these two lenses yielded almost the same focal length as that of the biconvex lens. The focal length $\mathrm{f}$ of a biconvex lens is given as follows [4]:

$$
\frac{1}{f}=(n-1)\left(\frac{1}{R_{1}}-\frac{1}{R_{2}}\right)
$$

Where $R_{1}$ and $R_{2}$ are the radii of curvature of the left and right surfaces of the lens. Since $R_{1}$ is $270 \mathrm{~mm}$, $R_{2}$ is $400 \mathrm{~mm}$, and $n$ is 1.504 , the focal length of the biconvex lens is $320 \mathrm{~mm}$ from Eq. (1). To measure the effective focal length $f_{\mathrm{e}}$ of the lens, which is limited by absorbing material, as shown in Fig. 3, $V$ (image distance) and $U$ (object distance) were measured first. Then by fixing the transmitter at $U=1500$ $\mathrm{mm}$, the single receiver was fixed on the stepping motor to measure the power distribution in two dimensions. For the power distribution measurement along the axis, the signal was measured in the range of 300-500 $\mathrm{mm}$ from the lens to receiver with a step of $10 \mathrm{~mm}$. For the power distribution perpendicular to the axis, the signal was measured in a $100 \mathrm{~mm}$ range with a step of $2 \mathrm{~mm}$.

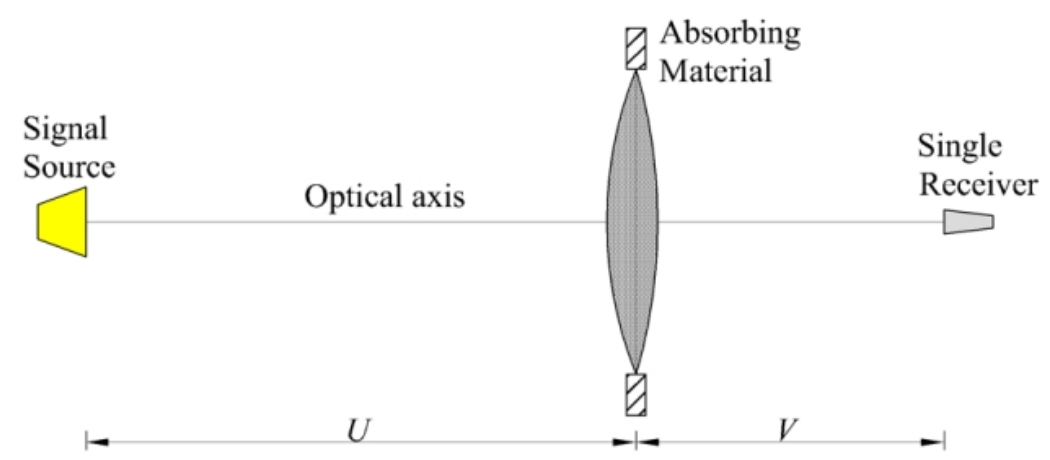

Figure 3. Schematic for measuring effective focal length

Fig. 4. shows a typical two-dimensional power distribution of a horn-lens combination. The intensity distribution on the optical axis is shown in Fig. 5. A power intensity maximum at $V=360 \mathrm{~mm}$ is clearly observed. The optimum image distance is obtained when the maximum output power of the receiver is obtained [16]. Therefore, the best image distance is $\mathrm{V}=360 \mathrm{~mm}$. 


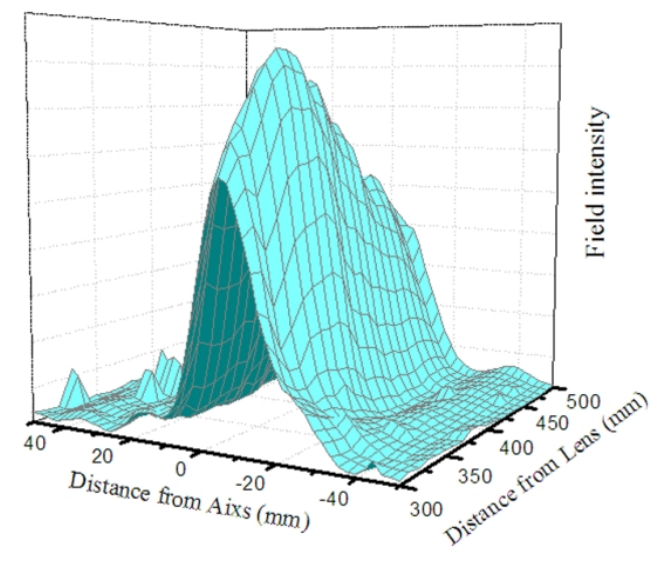

Fig. 4. Two-dimensional power distribution

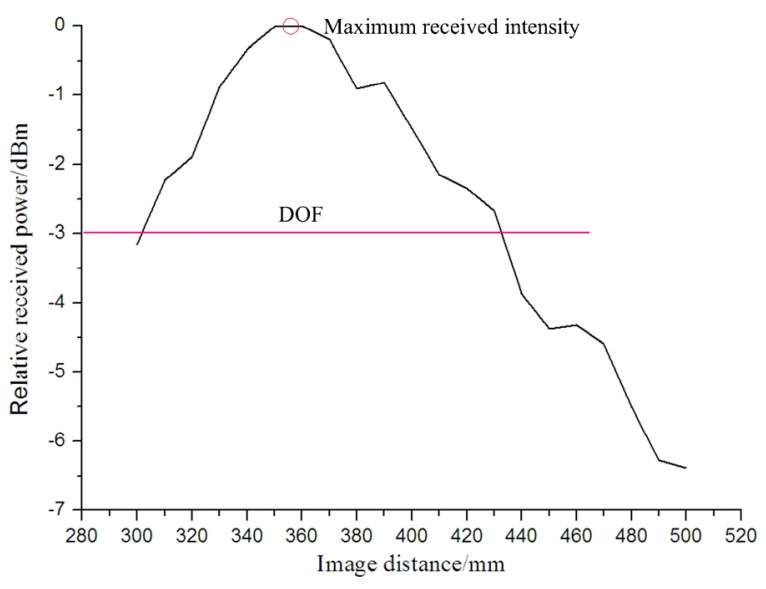

Fig. 5. Relative received power vs the image distance

The main characteristics of a lens are its beam spot size, spatial resolution (SR), and depth of focus (DOF) [16].The DOF of the lenses can be calculated by computing $-3 \mathrm{~dB}$ level ranges where the received power is $3 \mathrm{~dB}$ lower than the maximum power at the optimum image distance. The DOF, of $-3 \mathrm{~dB}$ ranges is around $130 \mathrm{~mm}$. The focal spot is the position that corresponds to where the power of the plane, which is perpendicular to the direction of propagation, drops to $3 \mathrm{~dB}$. In imaging applications, the SR is ultimately limited by diffraction. Calculating the maximum possible SR of a lens requires an arbitrary definition of what is meant by resolving two features. According to the Rayleigh criterion, the smallest resolvable distance is:

$$
r=\frac{1.22 \lambda(M+1) f}{D}
$$

where $M$ is the ratio of the image distance to the object distance, and $\lambda$ is the wavelength. $f_{\mathrm{e}}$ of the lens was calculated as $290 \mathrm{~mm}$ using the Gaussian image formula:

$$
\frac{1}{U}+\frac{1}{V}=\frac{1}{f_{\mathrm{e}}}
$$

$f_{\mathrm{e}}$ is slightly smaller than the theoretical focal length $f=320 \mathrm{~mm}$. This difference may be attributed to the fabrication and measurement errors. The effective focal length is in good agreement with the theoretical value, which demonstrates that the CGC material has a good refractive power on millimeter waves.

\section{Image test}

The schematic of the active millimeter-wave (AMMW) imaging system is shown in Fig. 6. The reflected power from the object illuminated by the signal generator is captured and focused on the image plane by the biconvex lens. The focused MMW is received by a single receiver that comprises a power diode, two low-noise amplifiers, and a low-pass filter.

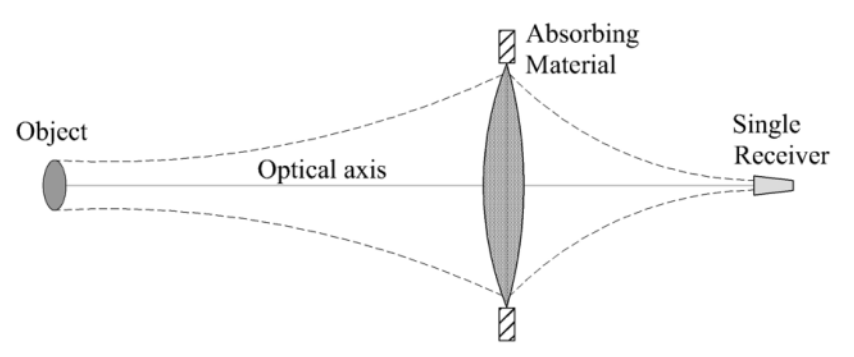

Fig. 6. Schematic of AMMW measurement system

The 2D images of the twin bars with different spacing and the square, which are made of tinfoil, were reconstructed by the AMMW measurement system when the objects were placed in an object 
plane $1280 \mathrm{~mm}$ away from the lens. The visible light images and the MMW images are shown in Figs. 7,8 and 9.
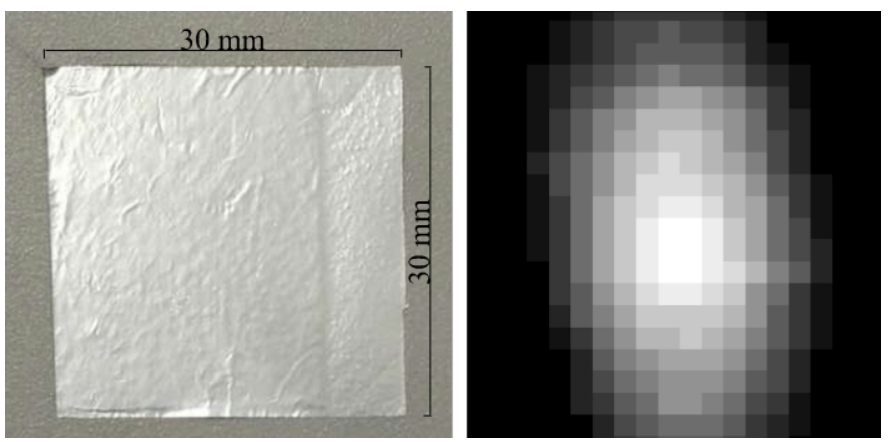

Fig. 7. Photo and MMW image of square
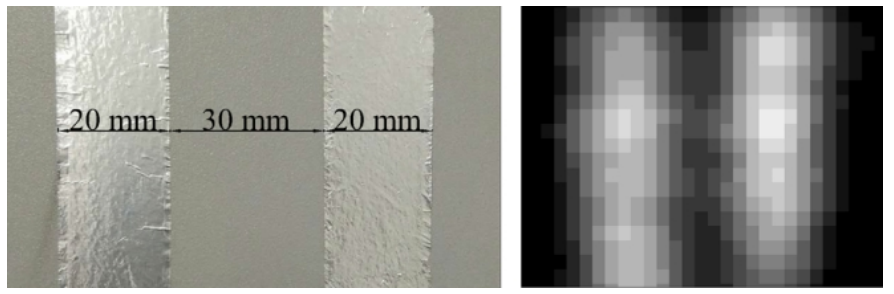

Fig. 8. Photo and MMW image of twin bars with a spacing of $30 \mathrm{~mm}$
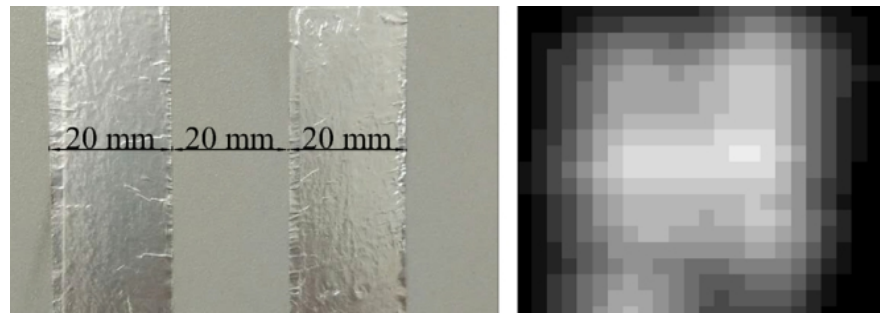

Fig. 9. Photo and MMW image of twin bars with a spacing of $20 \mathrm{~mm}$

The MMW image in Fig. 7 illustrates that the AMMW system based on the CGC material lens can recognize a $30 \times 30 \mathrm{~mm}^{2}$ square. We found that the MMW image was uneven in brightness, unclear $\mathrm{n}$ edges and oval-like in shape, which should be attributed to the uneven illumination of the horn source. Fig. 8 shows that the AMMW system can recognize the metal bars with a width of $20 \mathrm{~mm}$ and can distinguish the two bars with a spacing of $30 \mathrm{~mm}$. However, the system cannot distinguish the two bars with a spacing less than $20 \mathrm{~mm}$. According to Eq. (2), we calculate that the minimum theoretical size that the system can resolve is $19.4 \mathrm{~mm}$. The difference between the theoretical resolution and the experiment result should be attributed to the fact that in the Rayleigh criterion, the light source is the ideal point source and two bars are used in the experiment.

\section{Conclusions}

The biconvex lens made of CGC materials and PMMA materials has been designed, fabricated and measured. The refractive index of CGC is 1.504 at $35 \mathrm{GHz}$. The biconvex lens had a diameter of 200 $\mathrm{mm}$ and the radii of curvature on two sides are $270 \mathrm{~mm}$ and $400 \mathrm{~mm}$ respectively. In the system, the theoretical smallest resolvable distance and measured depth of focus are $19.4 \mathrm{~mm}$ and $130 \mathrm{~mm}$, respectively. The measured effective focal length is in good agreement with the theoretical value. The MMW images of the square and the twin bars made from tinfoil were acceptable. The AMMW system is capable of resolving two bars with a spacing of $30 \mathrm{~mm}$. The ability of the AMMW system proves that CGC materials have sufficient refracting power to MMW. 


\section{Acknowledgement}

This research was financially supported by the Beijing Education Committee Cooperation Building Foundation Project (110651101).

\section{References}

[1] M. R. Fetterman, J. Grata, G. Jubic, W. L. Kiser, and A. Visnansky, Simulation, acquisition and analysis of passive millimeter-wave images in remote sensing applications, Opt. Express 16 (2008) 20503-20515

[2] R. Appleby and R. N. Anderton, Millimeter-wave and submillimeter-wave imaging for security and Surveillance, Proc. IEEE 95 (2007) 1683-1690

[3] K. Yang, J. B. Wang, L. Zhao, Z. G. Liu, and T. Zhang, Millimeter-wave imaging with slab focusing lens made of electromagnetic-induction materials, Opt. Express 24 (2016) 566-572.

[4] J. B. Wang, H. X. Mei, K. Yang, L. Zhao, Z. G. Liu, and T. Zhang, Research on an artificial dielectric material for millimeter-wave imaging application, Appl. Opt. 56 (2017) 1947-1952.

[5] L. Y. Chang, C. C. Nien, C. M. Li, C. Y. Huang, Y. C. Yu, and J. H. Tarng, A simulation method of image construction for passive millimeter-wave imaging system, in IEEE 26th Convention of Electrical and Electronics Engineers in Israel (IEEEI) 26 (2010) pp. 713-716

[6] P. F. Goldsmith, Zone plate lens antennas for millimeter and submillimeter wavelengths, in The Third International Symposium on Space Terahertz Technology: Symposium Proceedings (1992) 345-361

[7] W. G. Kim, N. W. Moon, J. Kang, and Y. H. Kim, Loss measuring of large aperture quasi-optics for W-band imaging radiometer system, Prog. Electromagnetics Res. 125 (2012) 295-309.

[8] B. Schoenlinner, X. D. Wu, J. P. Ebling, G. V. Eleftheriades, and G. M. Rebeiz, Wide-scan spherical-lens antennas for automotive radars, IEEE Trans. Microw. Theory Tech. 50 (2002) 2166-2175

[9] T. Zhang, "Refraction of light in media, Sci. China Phys. Mech. 50 (2007) 591-600.

[10] M. Zhu, W. Liu, and T. Zhang, Calculation of electron wave functions and refractive index of Ne, Sci. China Phys. Mech. 51 (2008)1489-1495.

[11] G. Q. Xie, K. Ma, and S. Z. Huang, Theoretical calculation of the refractive index for Argon, J. Atom. Mol. Phys. 26 (2009) 817-820.

[12] Information on http://www.paper.edu.cn/releasepaper/content/200703-474

[13] Information on http://arxiv.org/abs/1409.7210

[14] Z. Zhuang, Research on Quasi-Optical Technique for Millimeter Wave Imaging (Harbin Institute of Technology, 2009)

[15] Lee J.J. Lens Antennas, In: Lo Y.T., Lee S.W. (Eds) Antenna Handbook, Springer, Boston, MA(1988)

[16] W. G. Kim, N. W. Moon, M. K. Singh, H. K. Kim, and Y. H. Kim, Characteristic analysis of aspheric quasi-optical lens antenna in millimeter-wave radiometer imaging system, Appl. Opt. 52 (2013) 1122-1131 